\title{
Proceedings of the Uehara Memorial Foundation. Innovative Medicine: Basic Research and Development. Cardiac Reprogramming for Heart Repair
}

\author{
Masaki Ieda
}

\begin{abstract}
Heart disease remains the leading cause of death worldwide. Terminally differentiated cardiomyocytes do not possess regenerative capacity, and heart disease is irreversible. Stem cell-derived cardiomyocytes are an attractive cell source for heart regeneration, but the risk of tumor formation due to contamination of stem cells, the complicated process of cell transplantation, and poor survival of the transplanted cells may be challenges for this approach. The discovery of reprogramming of fibroblasts into induced pluripotent stem cells (iPSCs) by the Yamanaka factors, Oct4, Sox2, Klf4, and c-Myc, inspired a new strategy to generate desired cell types from fibroblasts. It has been demonstrated that a diverse range of cell types, such as pancreatic $\beta$ cells, blood cells, neurons, chondrocytes, and hepatocytes, can be directly generated from fibroblasts, using lineage-specific transcription factors. We first reported that functional cardiomyocytes can be generated from mouse fibroblasts using cardiac-specific transcription factors, Gata4, Mef2c, and Tbx5 (GMT) in vitro. Our subsequent work revealed that GMT can also convert resident cardiac fibroblasts into cardiomyocyte-like cells in infarcted mouse hearts. We also demonstrated that Gata4, Mef2c, Tbx5, Myocd, and Mesp1 (GMTMM) can convert human fibroblasts into cardiomyocyte-like cells, and that addition of miR133 to GMT or GMTMM promoted cardiac reprogramming in mouse and human fibroblasts. Intriguingly, miR-133 directly suppressed Snai1, a master gene of epithelial-to-mesenchymal transition, which in turn repressed fibroblast signatures and promoted cardiac reprogramming. Here, I review the recent studies in cardiac reprogramming and discuss the perspectives and challenges of this innovative technology toward regenerative therapy.
\end{abstract}

\footnotetext{
M. Ieda, M.D., Ph. D. (ه)

Department of Clinical and Molecular Cardiovascular Research, Keio University School of Medicine, 35 Shinanomachi, Shinjuku-ku, Tokyo 160-8582, Japan

Department of Cardiology, Keio University School of Medicine, 35 Shinanomachi, Shinjuku-ku, Tokyo 160-8582, Japan
}

JST, CREST, 35 Shinanomachi, Shinjuku-ku, Tokyo 160-8582, Japan

e-mail: mieda@z8.keio.jp

K. Nakao et al. (eds.), Innovative Medicine, DOI 10.1007/978-4-431-55651-0_21 
Keywords Heart $\bullet$ Regeneration $\bullet$ Induced cardiomyocytes $\bullet$ Direct reprogramming $\bullet$ Transcription factors $\bullet$ miRNA

\section{Introduction}

Cardiovascular disease remains a leading cause of death worldwide for which current therapeutic regimens remain limited. As terminally differentiated cardiomyocytes have little regenerative capacity following injury, the demand for cardiac regenerative therapy is high. Following myocardial injury, endogenous cardiac fibroblasts, which account for more than half of the cells in the heart, proliferate and synthesize extracellular matrix, leading to fibrosis and heart failure. The large population of cardiac fibroblasts might be a potential source of cardiomyocytes for regenerative applications, if they could be reprogrammed into functional cardiomyocytes in situ.

The discovery of induced pluripotent stem cells (iPSCs) by Drs. Takahashi and Yamanaka provided a new strategy, which is direct generation of specific cell types from fibroblasts, using a combination of lineage-specific factors without mediating through a stem cell state. Recent studies demonstrated that direct reprogramming yields a diverse range of medically relevant cell types, including pancreatic $\beta$ cells, neurons, chondrocytes, and hepatocytes from fibroblasts [1-7]. Recently, we and others reported that cardiac and skin fibroblasts could be directly reprogrammed into cardiomyocyte-like cells by several combinations of cardiac-specific factors [8-12]. We discovered that a combination of three cardiac-specific transcription factors, Gata4, Mef $2 c$, and Tbx5, directly induced cardiomyocyte-like cells from mouse fibroblasts in vitro [8]. Subsequent studies revealed that direct gene transfer of the cardiac reprogramming factors in vivo could convert resident cardiac fibroblasts into cardiomyocytes in infarcted hearts, and improved cardiac function after myocardial infarction (MI) [12-14]. We and others also reported that human fibroblasts could be reprogrammed into cardiomyocyte-like cells by addition of cardiac transcription factors and miRNAs to GMT [15-17]. More recently, we found that cardiac-enriched miRNA, miR-133a (miR-133), promoted cardiac reprogramming in mouse embryonic fibroblasts (MEFs), adult mouse cardiac fibroblasts, and human cardiac fibroblasts (HCFs) in combination with GMT or GMTMM transduction. Mechanistically, miR-133 suppressed the fibroblast program by directly repressing Snai1, a master regulator of epithelial-to-mesenchymal transition (EMT), and thereby promoted cardiac reprogramming. This chapter will discuss recent studies of direct cardiac reprogramming using defined factors and provide a future perspective of this new technology. 


\section{Cell Fate Conversion by Transcription Factors}

John Gurdon and colleagues demonstrated that the nucleus of a differentiated frog cell, when transferred into an enucleated egg, could be converted to the totipotent zygote and give rise to a whole new frog [18]. Davis et al. found that $M y o D$, a master gene of skeletal muscle, converted fibroblasts into myoblasts [19-21]. Despite the race to identify single transcription factors that could guide cell fate similarly to MyoD for other lineages, including cardiomyocytes, the MyoD paradigm appeared to be an exception rather than the rule. Takahashi and Yamanaka achieved a breakthrough in this field by overexpressing four embryonic stem cell (ESC)-specific transcription factors in fibroblasts and generating pluripotent stem cells, iPS cells. Using retroviral vectors, they expressed 24 candidate genes and selected for reprogramming by expression of Fbx15, a gene specifically expressed in pluripotent stem cells. The combination of 24 factors induced formation of colonies with characteristic ESC morphology, and successive selection led to the minimally required core set of four genes, comprising Oct4, Sox2, Klf4, and c-Myc [22]. Many laboratories have since improved iPSC generation techniques to show that iPSCs share all features of ESCs, including expression of pluripotency markers, epigenetic marks, and the ability to generate chimeric mice.

The generation of iPSCs sparked a new idea, which is converting one cell type directly into another, using a combination of lineage-specific factors instead of single master genes. For example, Vierbuchen et al. converted mouse fibroblasts into functional neurons in vitro, using the neuronal lineage-specific transcription factors Ascll, Brn2, and Myt1l [4]. Zhou et al. provided the first evidence of cellular reprogramming in vivo using defined factors [5]. They showed that gene transfer of three transcription factors, $N g n 3, P d x 1$, and Mafa [23], efficiently reprogrammed pancreatic exocrine cells into functional $\beta$ cells in the mouse. The newly generated $\beta$ cells in vivo were indistinguishable from endogenous islet $\beta$ cells in terms of their structure and gene expression.

\section{Direct Reprogramming of Mouse Fibroblasts into Cardiomyocytes in Vitro}

In our study, we used cardiac fibroblasts (CFs) as a cell source for cardiac reprogramming. CFs are found throughout cardiac tissues along with cardiomyocytes, accounting for more than half of the cells in the heart, and may be a potential cell source for cardiac repair. To determine the candidate factors of cardiac reprogramming, we identified the genes that are specifically expressed in embryonic cardiomyocytes. We developed a novel cell purification system in which embryonic cardiomyocytes and CFs can be purified using FACS [24]. Using this system, we selected 14 factors as candidates for cardiac reprogramming, which are specifically expressed in cardiomyocytes and are critical for heart development. 
We next developed a screening system in which the induction of mature cardiomyocytes from fibroblasts could be analyzed quantitatively by FACS. We generated $\alpha$ myosin heavy chain $(\alpha M H C)$ promoter-driven EGFP-IRES-Puromycin transgenic mice $(\alpha \mathrm{MHC}-\mathrm{GFP})$, in which only mature cardiomyocytes expressed green fluorescent protein (GFP) $[8,25,26]$. Transduction of all 14 factors into fibroblasts induced $1.7 \%$ of $\mathrm{GFP}^{+}$cells, and serial removal of individual factors demonstrated that a combination of three factors (Gata4, Mef2c, and Tbx5) were sufficient for efficient $\mathrm{GFP}^{+}$cell induction (around $15 \%$ ) from CFs. The three cardiac reprogramming factors, Gata4, Mef2c, and Tbx5, are core cardiac transcription factors during early heart development [27-29] and are known to interact with each other, coactivate cardiac gene expression, and promote cardiomyocyte differentiation [30-32]. We designated these $\mathrm{GFP}^{+}$cardiomyocyte-like cells as induced cardiomyocytes (iCMs). The iCMs expressed several cardiac marker proteins, such as sarcomeric $\alpha$-actinin, cardiac troponin T, and atrial natriuretic factor (Fig. 1). They also had well-defined sarcomeric structures similar to neonatal cardiomyocytes. The global gene expression profile of $\mathrm{GFP}^{+} \mathrm{iCMs}$ is not identical but similar to neonatal cardiomyocytes, and different from original CFs. We also found that functionally important cardiac genes, such as ion channel and sarcomere genes, were upregulated more in 4-week iCMs than in 2-week iCMs, suggesting that cardiac differentiation occurred over several weeks. The chromatin state of iCMs was also similar to cardiomyocytes but different from fibroblasts in histone modifications and DNA methylation patterns [8]. These results suggest that iCMs are cardiomyocyte-like cells in gene expression and epigenetic states. We also demonstrated that iCMs possessed functional properties characteristic of cardiomyocytes. The iCMs exhibited intracellular $\mathrm{Ca}^{2+}$ transient and action potentials after 2-4 weeks of culture. In addition, CF-derived iCMs contracted spontaneously. To determine if the iCMs could arise from cells in other organs, we transduced the three factors into mouse tail-tip fibroblasts (TTFs). TTF-derived iCMs expressed cardiac markers and

a

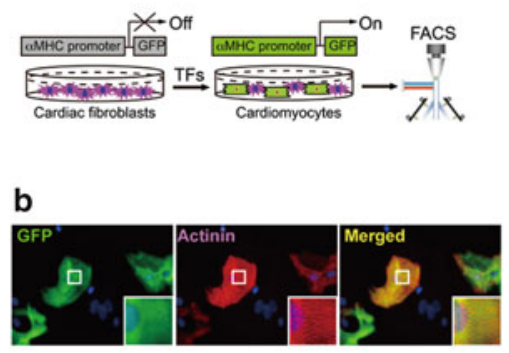

C

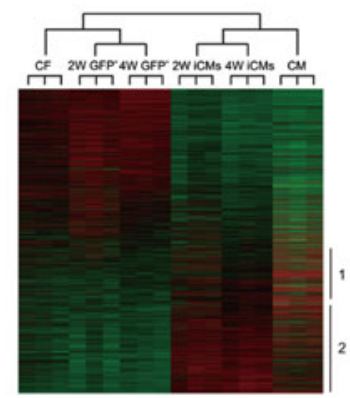

d

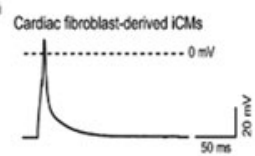

Adut mouse ventricular cardicmyocyies

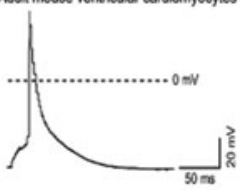

Fig. 1 Cardiac reprogramming in mouse fibroblasts. (a) Screening system for cardiac reprogramming factors. The $\alpha$-MHC-GFP turns on, if reprogramming succeeds. (b) Immunohistochemistry for iCMs. Mouse fibroblasts were converted into cardiomyocyte-like cells (iCMs) by transduction of Gata4, Mef2c, and Tbx5. The iCMs expressed cardiac proteins and had striated muscle structures. (c) Microarray analyses for fibroblasts, iCMs, and cardiomyocytes. (d)Action potential recordings from iCMs and cardiomyocytes (Cited from Ieda et al. [8]) 
spontaneous intracellular $\mathrm{Ca}^{2+}$ transients, while the percentage of cardiac troponin $\mathrm{T}^{+}$cells was $50 \%$ less than that of CF-derived iCMs. The TTF-derived iCMs had some functional characteristics of cardiomyocytes, such as spontaneous intracellular $\mathrm{Ca}^{2+}$ transients, but we did not observe cellular contraction, suggesting that TTFs are more resistant to cardiac reprogramming by GMT. These findings exclude the possibility that iCMs arise from contamination of cardiomyocytes or cardiac progenitors in the fibroblast population [33, 34].

While direct conversion of somatic cells to $\beta$ cells and neurons was reported, the "route" of cell fate change was not clear. There are two possibilities for the reprogramming of fibroblasts into differentiated cardiomyocytes. One is direct conversion of fibroblasts into differentiated cardiomyocytes, and the other is that fibroblasts first revert to a cardiac progenitor/stem cell state before further cardiac differentiation. We were able to genetically test these two possibilities by using mice expressing Isl1Cre-yellow fluorescent protein (YFP) and Mesp1-Cre-YFP obtained by crossing Isl1-Cre or Mesp1-Cre mice with R26R-EYFP mice [35]. Isl1 and Mesp1 are transiently expressed in the early cardiac progenitor cells before further cardiac differentiation $[36,37]$. We found that iCMs induced from Isl1-Cre-YFP and Mesp1-Cre-YFP fibroblasts did not express YFP, suggesting that the fibroblasts directly reprogrammed into differentiated cardiomyocytes without passing through a cardiac progenitor cell state. We next investigated whether GMT-transduced CFs can be transplanted in vivo and be reprogrammed into cardiomyocytes within the heart. CFs infected with GMT expressed $\alpha$-MHC GFP and sarcomeric markers, and converted into cardiomyocytelike cells in the mouse hearts after 2 weeks of cell transplantation.

Following our initial report, other groups also reported generation of cardiomyocyte-like cells from mouse fibroblasts based on the same factors and microRNAs. Song et al. reported that adding Hand2 to GMT converted adult CFs and TTFs into functional cardiomyocyte-like cells more efficiently than GMT alone [12]. Protze et al. found that the combination of Mef2c, myocardin, and Tbx5 upregulated a broad spectrum of cardiac genes and induced functional cardiomyocytes from MEFs and CFs [11]. Jayawardena et al. reported that a combination of muscle-specific microRNAs, mir-1, 133, 208, and 499, can convert CFs into functional cardiomyocyte-like cells [10]. Thus, several combinations of cardiogenic factors can induce cardiac reprogramming, which is similar to the experience in the iPSC field. Although the induction of fully matured cardiac cells remains low in culture, and further refinements are needed, these results suggest that fibroblasts transduced with cardiac reprogramming factors ex vivo can be delivered to damaged myocardium for regenerative purposes.

\section{Cardiac Reprogramming in Vivo by Gene Transfer of Defined Factors}

On the basis of the findings of direct cardiac reprogramming in vitro, we investigated whether gene transfer of GMT into mouse injured hearts could similarly induce new cardiomyocyte generation [14]. Transduction of GMT retroviruses 
converted adult CFs from infarcted hearts into iCMs in vitro. Injection of GMT retroviruses into $\alpha \mathrm{MHC}-\mathrm{GFP}$ transgenic mouse hearts induced the expression of $\alpha \mathrm{MHC}-\mathrm{GFP}$, a reporter of cardiomyocytes, in $3 \%$ of virus-infected fibroblasts. A mixture of GMT injection into the immunosuppressed mouse hearts induced cardiac protein expression in retrovirus-infected cells within 2 weeks, with a conversion rate of approximately $1 \%$, although few cells showed striated muscle structures. Next, to transduce GMT more efficiently in vivo, we developed a new polycistronic retrovirus vector expressing GMT separated by $2 \mathrm{~A}$ "self-cleaving" peptides (3F2A). In vivo gene transfer of $3 \mathrm{~F} 2 \mathrm{~A}$ into infarcted hearts resulted in generation of $\mathrm{iCMs}$ in fibrotic tissues, which expressed sarcomeric $\alpha$-actinin, cardiac troponin $\mathrm{T}$, and several cardiac-specific genes, and had clear cross-striations. These results suggest that $3 \mathrm{~F} 2 \mathrm{~A}-\mathrm{iCMs}$ are more mature cardiomyocytes, and that the polycistronic vector can be used for cellular reprogramming in vivo.

In parallel with our study, two other groups also published reports on in vivo cardiac reprogramming in infarcted mouse hearts. Qian et al. found that fibroblasts in infarcted hearts were converted into cardiomyocyte-like cells by GMT retroviral gene transfer [13]. Song et al. reported that adding Hand2 to GMT converted endogenous CFs into functional cardiomyocyte-like cells more efficiently than GMT alone in vivo [12]. Both studies demonstrated that the in vivo iCMs had wellorganized sarcomeric structures and exhibited functional characteristics of adult ventricular cardiomyocytes, including cellular contraction, electrophysiological properties, and functional coupling to other cardiac cells. They also demonstrated that retroviral gene transfer of reprogramming factors into infarcted hearts significantly improved cardiac function and reduced fibrosis 2 and 3 months after myocardial infarction. Although all three studies, including ours, demonstrated in vivo cardiac reprogramming, the approaches used to address this issue differed. The other two groups used mainly fibroblast-lineage tracing mice to demonstrate cardiac conversion from CFs, while we co-transduced GMT with marker genes (GFP or DsRed) to demonstrate cardiac induction in the infected fibroblasts, using $\alpha \mathrm{MHC}-\mathrm{GFP}$ transgenic and nude mice. Although it is not clear how many newly generated iCMs remained in the injured hearts in the longer-term follow-up, and to what extent the iCMs contributed to the improvement in cardiac function, these findings might inform new regenerative strategies for repairing injured hearts. Further work in larger animals and more efficient gene delivery system in vivo are needed for future research.

\section{Gata4/Mef2c/Tbx5/Myocd/Mesp1 Reprogram Human Fibroblasts into Cardiomyocyte-Like Cells}

We next analyzed whether human fibroblasts could be directly converted to iCMs by defined factors [17]. We found that GMT was not sufficient for cardiac induction in HCFs. Thus, we screened additional factors that enhance reprogramming, and 
found that addition of Mesp1 and Myocd to GMT upregulated a broader spectrum of cardiac genes more efficiently than GMT alone. The HCFs and human dermal fibroblasts transduced with GMT, Mesp1, and Myocd (GMTMM) changed the cell morphology to a rod-like or polygonal shape, expressed a broad range of cardiac genes and concomitantly suppressed fibroblast genes, and exhibited spontaneous $\mathrm{Ca}^{2+}$ oscillations. Moreover, the cells matured to exhibit action potentials and contract synchronously in coculture with murine cardiomyocytes. The EdU assay revealed that the human iCMs did not pass through a mitotic stem cell state and were directly generated from fibroblasts.

Human cardiac reprogramming was also reported by other groups. Nam et al. reported that a combination of Gata4, Hand2, Tbx5, Myocd, miR-1, and miR-133 induced $13 \%$ of adult HCFs to express cardiac troponin T protein and that a small subset of the iCMs exhibited spontaneous contractility after 11 weeks in culture [15]. Islas et al. reported that transient overexpression of Ets2 and Mesp1 could reprogram human dermal fibroblasts into cardiac progenitor-like cells [16]. The induced cardiac progenitor-like cells differentiated into immature cardiomyocytes and exhibited calcium activities. These findings may represent an important initial step toward potential therapeutic applications of the direct reprogramming approach in clinical situations. However, human cardiac reprogramming process is slower and less efficient than mouse reprogramming, much like the induction of human iPSCs compared with mouse iPSCs. Future studies are needed to thoroughly optimize conditions for human cardiomyocyte generation and maturation and to characterize the properties of human iCMs.

\section{MiR-133 Promotes Cardiac Reprogramming by Repressing Snai1 and Silencing the Fibroblast Profile}

As discussed, induction of functional cardiomyocytes in vitro was inefficient and molecular mechanisms of direct reprogramming remained undefined. In contrast, the in vivo iCMs were more fully reprogrammed than their counterparts in vitro, suggesting the presence of undefined factors that may enhance reprogramming. Identification of such potent reprogramming factors could provide new insights into the mechanisms of cardiac reprogramming. We selected four cardiac miRNAs as candidate factors, which are specifically expressed in cardiomyocytes and skeletal muscles and are important for heart development and physiology. FACS analyses using $\alpha$ MHC-GFP mice revealed that these miRNAs alone did not reprogram MEFs into iCMs, but addition of miR-133 to GMT strongly enhanced cardiac induction compared with other miRNAs. The time courses of reprogramming between GMT and GMT/miR-133 revealed that the GMT/miR-133-iCMs showed sarcomeric structures after 7 days of infection, which normally takes 2 weeks with GMT alone. Moreover, cell contraction started from 10 days after GMT/miR-133 transduction, which generally took 4 weeks, and the number of beating cells increased over time, 
with sevenfold more contractile cells achieved compared with GMT alone. The global gene expression profiles of iCMs induced with GMT or GMT/miR-133 revealed that among 23,474 probes, 46 cardiac-enriched genes were upregulated and 129 fibroblast-related genes were downregulated by GMT/miR-133 induction. Molecularly, we found that Snai1, a basic helix-loop-helix transcription factor and a master regulator of EMT, was a direct target of miR-133 and had two conserved miR-133-binding sites within the 3' UTR. Snail knockdown suppressed fibroblast genes, upregulated cardiac genes, and induced more contracting iCMs in combination with GMT transduction, recapitulating the effects of miR-133 overexpression. In contrast, overexpression of Snail in GMT/miR-133-transduced cells activated the fibroblast signature and inhibited generation of beating iCMs. Moreover, miR133-mediated Snai1 repression was also critical for cardiac reprogramming in adult mice and HCFs in combination with GMT or GMTMM. These results are consistent with the cell fate conversion, in which the target cell program is progressively activated and the starting-cell profile is concomitantly suppressed during reprogramming. Although further work in identifying more efficient protocols and understanding molecular mechanisms of cardiac reprogramming are needed, these reports demonstrate that the cardiac reprogramming strategy might be a potential approach for heart regeneration in the future [38].

\section{Perspectives and Challenges of Cardiac Reprogramming for Clinical Applications}

As discussed above, the cardiac reprogramming field has extensively progressed and may change regenerative medicine in the future. The directly induced cardiac cells appear to quickly exit the cell cycle following the lineage conversion, and the utility of iCMs in vitro might be limited. Alternatively, direct induction of cardiac progenitor cells, as shown in neural stem/progenitor cell reprogramming, may be a good approach to solve this issue [39]. In contrast, introduction of cardiac reprogramming factors directly into the damaged heart may convert the endogenous cardiac fibroblast population, which represents $>50 \%$ of cardiac cells, into new functional cardiomyocytes in situ, and may improve cardiac function. This in vivo reprogramming approach may have several advantages compared with cell transplantation-based regeneration (Fig. 2). First, the process is simple and short; second, avoiding the reprogramming of pluripotent cells before cardiac differentiation would greatly lower the risk of tumor formation; and third, direct injection of defined factors obviates the need for cell transplantation, for which long-term cardiac cell survival remains a challenge.

Although direct cardiac induction using defined factors has been demonstrated by several laboratories, the reprogramming efficiency remains low and many pitfalls remain that may lead to reprogramming failure [40, 41]. Future studies will be needed to thoroughly optimize the protocol for iCM generation, and to characterize 


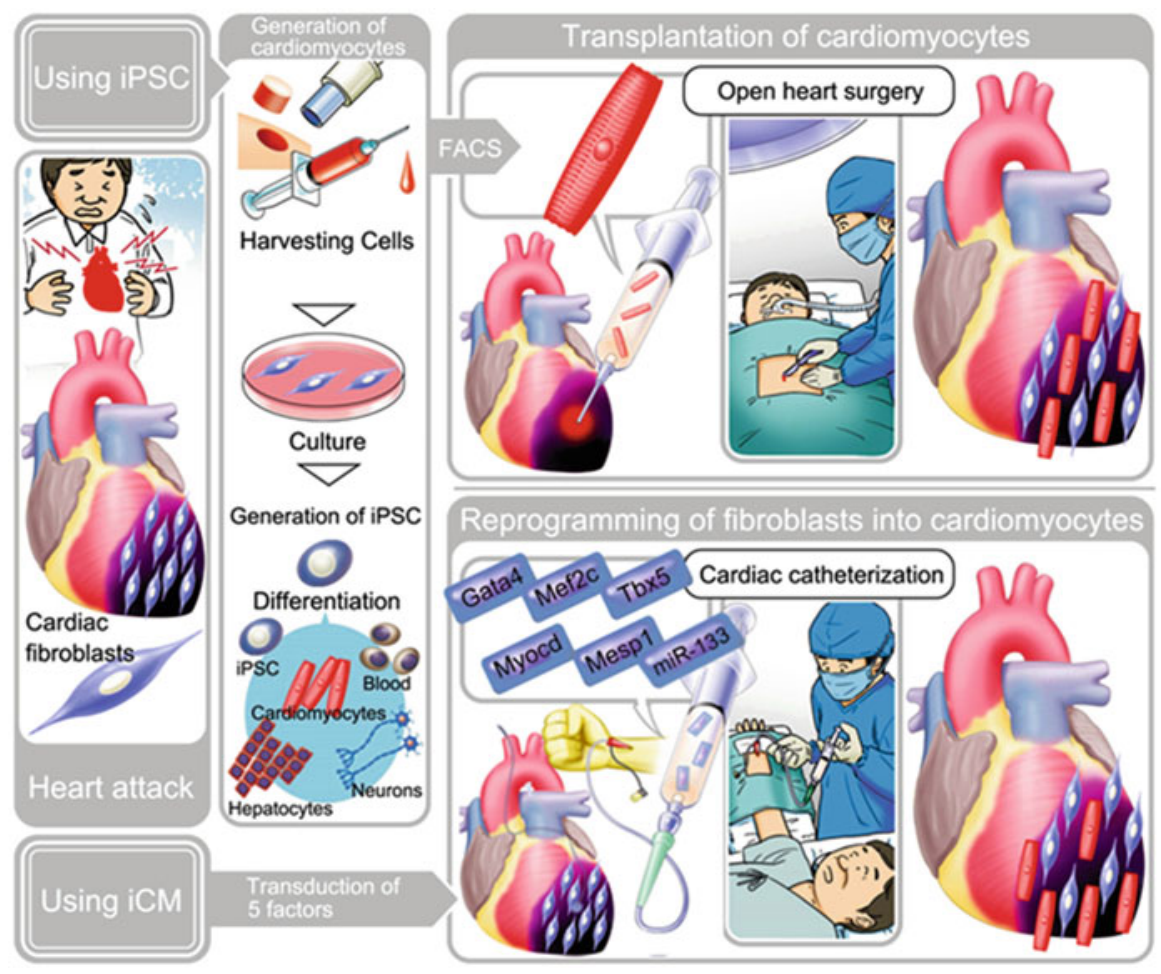

Fig. 2 Future heart regenerative therapies. The upper panel indicates the cell transplantation strategy using iPSC-derived cardiomyocytes to repair the damaged heart. The lower panel indicates the direct cardiac reprogramming strategy, which converts endogenous cardiac fibroblasts into iCMs in situ using defined factors

the properties of iCMs. Given that secreted proteins, electrical and mechanical stimulation, and cell-to-cell contact might promote cardiac differentiation and reprogramming in our experience, the future of this new technology seems to be bright and promising. Highly standardized protocols that make the process more efficient and more easily transferable between different laboratories should be developed in the future to push this field forward.

\section{Conclusions}

Cellular reprogramming has long been recognized as a possibility, although the impact of cell type conversion by defined factors was most prominently exemplified only recently by the discovery of iPSCs. This landmark finding fundamentally altered approaches to regenerative medicine, and provided a broad strategy to induce desired cell types by introducing lineage-specific factors. Detailed analyses 
of the properties of generated cells and understanding of the molecular mechanisms of reprogramming might be necessary to advance this nascent technology for future clinical applications.

Acknowledgments The author is grateful to the members of the Ieda Lab and many other labs for their critical work and discussions. The author apologizes to other authors whose work has not been cited, because of space limitations. The author was supported by a research grant from The Uehara Memorial Foundation for this paper.

\section{Disclosures None.}

Open Access This chapter is distributed under the terms of the Creative Commons Attribution Noncommercial License, which permits any noncommercial use, distribution, and reproduction in any medium, provided the original author(s) and source are credited.

\section{References}

1. Han DW, Tapia N, Hermann A, Hemmer K, Hoing S, Arauzo-Bravo MJ, Zaehres H, Wu G, Frank S, Moritz S, Greber B, Yang JH, Lee HT, Schwamborn JC, Storch A, Scholer HR (2012) Direct reprogramming of fibroblasts into neural stem cells by defined factors. Cell Stem Cell 10:465-472

2. Huang P, He Z, Ji S, Sun H, Xiang D, Liu C, Hu Y, Wang X, Hui L (2011) Induction of functional hepatocyte-like cells from mouse fibroblasts by defined factors. Nature 475:386-389

3. Marro S, Pang ZP, Yang N, Tsai MC, Qu K, Chang HY, Sudhof TC, Wernig M (2011) Direct lineage conversion of terminally differentiated hepatocytes to functional neurons. Cell Stem Cell 9:374-382

4. Vierbuchen T, Ostermeier A, Pang ZP, Kokubu Y, Sudhof TC, Wernig M (2010) Direct conversion of fibroblasts to functional neurons by defined factors. Nature 463:1035-1041

5. Zhou Q, Brown J, Kanarek A, Rajagopal J, Melton DA (2008) In vivo reprogramming of adult pancreatic exocrine cells to beta-cells. Nature 455:627-632

6. Hiramatsu K, Sasagawa S, Outani H, Nakagawa K, Yoshikawa H, Tsumaki N (2011) Generation of hyaline cartilaginous tissue from mouse adult dermal fibroblast culture by defined factors. J Clin Invest 121:640-657

7. Sekiya S, Suzuki A (2011) Direct conversion of mouse fibroblasts to hepatocyte-like cells by defined factors. Nature 475:390-393

8. Ieda M, Fu JD, Delgado-Olguin P, Vedantham V, Hayashi Y, Bruneau BG, Srivastava D (2010) Direct reprogramming of fibroblasts into functional cardiomyocytes by defined factors. Cell $142: 375-386$

9. Efe JA, Hilcove S, Kim J, Zhou H, Ouyang K, Wang G, Chen J, Ding S (2011) Conversion of mouse fibroblasts into cardiomyocytes using a direct reprogramming strategy. Nat Cell Biol $13: 215-222$

10. Jayawardena TM, Egemnazarov B, Finch EA, Zhang L, Payne JA, Pandya K, Zhang Z, Rosenberg P, Mirotsou M, Dzau VJ (2012) MicroRNA-mediated in vitro and in vivo direct reprogramming of cardiac fibroblasts to cardiomyocytes. Circ Res 110:1465-1473

11. Protze S, Khattak S, Poulet C, Lindemann D, Tanaka EM, Ravens U (2012) A new approach to transcription factor screening for reprogramming of fibroblasts to cardiomyocyte-like cells. J Mol Cell Cardiol 53:323-332

12. Song K, Nam YJ, Luo X, Qi X, Tan W, Huang GN, Acharya A, Smith CL, Tallquist MD, Neilson EG, Hill JA, Bassel-Duby R, Olson EN (2012) Heart repair by reprogramming nonmyocytes with cardiac transcription factors. Nature 485:599-604 
13. Qian L, Huang Y, Spencer CI, Foley A, Vedantham V, Liu L, Conway SJ, Fu JD, Srivastava D (2012) In vivo reprogramming of murine cardiac fibroblasts into induced cardiomyocytes. Nature 485:593-598

14. Inagawa K, Miyamoto K, Yamakawa H, Muraoka N, Sadahiro T, Umei T, Wada R, Katsumata Y, Kaneda R, Nakade K, Kurihara C, Obata Y, Miyake K, Fukuda K, Ieda M (2012) Induction of cardiomyocyte-like cells in infarct hearts by gene transfer of Gata4, Mef2c, and Tbx5. Circ Res 111:1147-1156

15. Nam YJ, Song K, Luo X, Daniel E, Lambeth K, West K, Hill JA, Dimaio JM, Baker LA, Bassel-Duby R, Olson EN (2013) Reprogramming of human fibroblasts toward a cardiac fate. Proc Natl Acad Sci U S A 110:5588-5593

16. Islas JF, Liu Y, Weng KC, Robertson MJ, Zhang S, Prejusa A, Harger J, Tikhomirova D, Chopra M, Iyer D, Mercola M, Oshima RG, Willerson JT, Potaman VN, Schwartz RJ (2012) Transcription factors ETS2 and MESP1 transdifferentiate human dermal fibroblasts into cardiac progenitors. Proc Natl Acad Sci U S A 109:13016-13021

17. Wada R, Muraoka N, Inagawa K, Yamakawa H, Miyamoto K, Sadahiro T, Umei T, Kaneda R, Suzuki T, Kamiya K, Tohyama S, Yuasa S, Kokaji K, Aeba R, Yozu R, Yamagishi H, Kitamura T, Fukuda K, Ieda M (2013) Induction of human cardiomyocyte-like cells from fibroblasts by defined factors. Proc Natl Acad Sci U S A 110:12667-12672

18. Gurdon JB, Elsdale TR, Fischberg M (1958) Sexually mature individuals of Xenopus laevis from the transplantation of single somatic nuclei. Nature 182:64-65

19. Berkes CA, Tapscott SJ (2005) MyoD and the transcriptional control of myogenesis. Semin Cell Dev Biol 16:585-595

20. Tapscott SJ (2005) The circuitry of a master switch: Myod and the regulation of skeletal muscle gene transcription. Development 132:2685-2695

21. Davis RL, Weintraub H, Lassar AB (1987) Expression of a single transfected cDNA converts fibroblasts to myoblasts. Cell 51:987-1000

22. Takahashi K, Yamanaka S (2006) Induction of pluripotent stem cells from mouse embryonic and adult fibroblast cultures by defined factors. Cell 126:663-676

23. Zhou Q, Law AC, Rajagopal J, Anderson WJ, Gray PA, Melton DA (2007) A multipotent progenitor domain guides pancreatic organogenesis. Dev Cell 13:103-114

24. Ieda M, Tsuchihashi T, Ivey KN, Ross RS, Hong TT, Shaw RM, Srivastava D (2009) Cardiac fibroblasts regulate myocardial proliferation through betal integrin signaling. Dev Cell $16: 233-244$

25. Ieda M, Kanazawa H, Kimura K, Hattori F, Ieda Y, Taniguchi M, Lee JK, Matsumura K, Tomita Y, Miyoshi S, Shimoda K, Makino S, Sano M, Kodama I, Ogawa S, Fukuda K (2007) Sema3a maintains normal heart rhythm through sympathetic innervation patterning. Nat Med 13:604-612

26. Gulick J, Subramaniam A, Neumann J, Robbins J (1991) Isolation and characterization of the mouse cardiac myosin heavy chain genes. J Biol Chem 266:9180-9185

27. Zhao R, Watt AJ, Battle MA, Li J, Bondow BJ, Duncan SA (2008) Loss of both GATA4 and GATA6 blocks cardiac myocyte differentiation and results in acardia in mice. Dev Biol 317:614-619

28. Srivastava D (2006) Making or breaking the heart: from lineage determination to morphogenesis. Cell 126:1037-1048

29. Olson EN (2006) Gene regulatory networks in the evolution and development of the heart. Science 313:1922-1927

30. Ghosh TK, Song FF, Packham EA, Buxton S, Robinson TE, Ronksley J, Self T, Bonser AJ, Brook JD (2009) Physical interaction between TBX5 and MEF2C is required for early heart development. Mol Cell Biol 29:2205-2218

31. Garg V, Kathiriya IS, Barnes R, Schluterman MK, King IN, Butler CA, Rothrock CR, Eapen RS, Hirayama-Yamada K, Joo K, Matsuoka R, Cohen JC, Srivastava D (2003) GATA4 mutations cause human congenital heart defects and reveal an interaction with TBX5. Nature 424:443-447 
32. Morin S, Charron F, Robitaille L, Nemer M (2000) GATA-dependent recruitment of MEF2 proteins to target promoters. Embo J 19:2046-2055

33. Andersen DC, Andersen P, Schneider M, Jensen HB, Sheikh SP (2009) Murine "cardiospheres" are not a source of stem cells with cardiomyogenic potential. Stem Cells 27:1571-1581

34. Davis DR, Zhang Y, Smith RR, Cheng K, Terrovitis J, Malliaras K, Li TS, White A, Makkar R, Marban E (2009) Validation of the cardiosphere method to culture cardiac progenitor cells from myocardial tissue. PLoS One 4:e7195

35. Srinivas S, Watanabe T, Lin CS, William CM, Tanabe Y, Jessell TM, Costantini F (2001) Cre reporter strains produced by targeted insertion of EYFP and ECFP into the ROSA26 locus. BMC Dev Biol 1:4

36. Bondue A, Lapouge G, Paulissen C, Semeraro C, Iacovino M, Kyba M, Blanpain C (2008) Mesp1 acts as a master regulator of multipotent cardiovascular progenitor specification. Cell Stem Cell 3:69-84

37. Laugwitz KL, Moretti A, Lam J, Gruber P, Chen Y, Woodard S, Lin LZ, Cai CL, Lu MM, Reth M, Platoshyn O, Yuan JX, Evans S, Chien KR (2005) Postnatal is11+ cardioblasts enter fully differentiated cardiomyocyte lineages. Nature 433:647-653

38. Nam YJ, Song K, Olson EN (2013) Heart repair by cardiac reprogramming. Nat Med 19:413-415

39. Lujan E, Chanda S, Ahlenius H, Sudhof TC, Wernig M (2012) Direct conversion of mouse fibroblasts to self-renewing, tripotent neural precursor cells. Proc Natl Acad Sci U S A 109:2527-2532

40. Chen JX, Krane M, Deutsch MA, Wang L, Rav-Acha M, Gregoire S, Engels MC, Rajarajan K, Karra R, Abel ED, Wu JC, Milan D, Wu SM (2012) Inefficient reprogramming of fibroblasts into cardiomyocytes using gata4, mef2c, and tbx5. Circ Res 111:50-55

41. Srivastava D, Ieda M (2012) Critical factors for cardiac reprogramming. Circ Res 111:5-8 